\title{
Effects of Asymmetric Electrical Pulse on Retinal Excitement for Retinal Prostheses
}

\author{
Yukari Nakano, ${ }^{1,2 *}$ Yasuo Terasawa, ${ }^{1,2}$ Hiroyuki Kanda, ${ }^{3}$ \\ Jun Ohta, ${ }^{2}$ Hajime Sawai, ${ }^{4}$ and Takashi Fujikado ${ }^{3}$ \\ ${ }^{1}$ Artificial Vision Institute, Research and Development Division, Nidek Co., Ltd., \\ 13-2 Hama-cho, Gamagori, Aichi 443-0036, Japan \\ ${ }^{2}$ Graduate School of Materials Science, Nara Institute of Science and Technology, \\ 8916-5 Takayama-cho, Ikoma, Nara 630-0192, Japan \\ ${ }^{3}$ Department of Applied Visual Science, Osaka University Graduate School of Medicine, \\ 2-2 Yamadaoka, Suita, Osaka 565-0871, Japan \\ ${ }^{4}$ Department of Health Sciences, School of Nursing, Osaka Prefecture University, \\ 3-7-30 Habikino, Habikino, Osaka 583-8555, Japan
}

(Received July 21, 2017; accepted December 8, 2017)

Keywords: retinal prosthesis, asymmetric pulse, electrical stimulation parameter, retinitis pigmentosa

Retinal prostheses for blindness electrically stimulate retinas with photoreceptor cell degeneration to evoke a pseudolight sensation (phosphenes). Biphasic symmetric pulses with charge balancing are commonly used for electrical stimulation in retinal prostheses. It has been reported that a charge-balanced biphasic asymmetric pulse can reduce the threshold for electrical evoked responses in cochlear implants. In this study, in order to determine the effectiveness of an asymmetric pulse, we applied single biphasic symmetric and asymmetric pulses transretinally to the eyeball of anesthetized rats. We found that the application of an anodal pulse with a shorter duration and a higher current amplitude evoked a large field response in the superior colliculus (SC) in comparison with a symmetric pulse with the same electrical charge. This result suggests that an asymmetric pulse with a short pulse duration and high current amplitude in the anodal phase is more effective for retinal prostheses than a symmetrical pulse with the same electrical charge.

\section{Introduction}

In recent years, engineering-based research on attempting to utilize the brain has advanced rapidly. One field of such research is devices and technologies called brain-machine interfaces (BMIs), which artificially connect the brain and machines directly and enable them to interact with each other. BMIs are classified into two major categories, output BMIs and input BMIs. ${ }^{(1)}$ Output BMIs can decode the neural outputs from the brain to control devices, such as a robotic arm. ${ }^{(2)}$ In contrast, input BMIs are used for sensory disabilities, such as blindness and deafness, and their sensor devices can receive external signals to provide the sensory nervous system with the corresponding inputs. Cochlear implants are a representative example of an input BMI and *Corresponding author: e-mail: yukari_nakano@nidek.co.jp http://dx.doi.org/10.18494/SAM.2018.1701 
have been used to reconstruct the hearing of many people worldwide. ${ }^{(3)}$ The visual equivalent of a cochlear implant is a retinal prosthesis for blindness caused by photoreceptor degeneration, such as retinitis pigmentosa (RP). It is a genetic disease associated with the slow progressive impairment of vision and ultimately leads to blindness, having an incidence of 1 in 4000 people worldwide. $^{(4)}$ At present, no fundamental therapy for RP has been established, but retinal prostheses have been studied as a means of restoring the lost visual function in individuals with this condition. Retinal prostheses have been put into practical use in the United States and Europe in recent years. ${ }^{(5,6)}$

The prostheses are primarily composed of a camera, an image processor, and an electrical stimulator with a microelectrode array (MEA). The camera can capture visual images as alternatives to retinal photoreceptors, and the processor can convert the images into digital commands for stimulating the degenerated retina by the stimulator implanted in the eye globe of patients with RP. The stimulation through the MEA can activate the residual retinal circuitry, generating pseudolight sensations (phosphenes). Currently, there are at least three types of MEA implantation: epiretinal (MEA on the retina from the vitreous side) ${ }^{(5)}$ subretinal (between the choroid and retina), ${ }^{(6)}$ and suprachoroidal/subscleral (between the choroid and retinal pigment epithelium, ${ }^{(7)}$ or in the sclera ${ }^{(8)}$ ). We have been developing a retinal prosthesis based on suprachoroidal transretinal stimulation (STS), where the electrode array is positioned in a scleral pocket created by a half-layer incision. ${ }^{(8)}$ The advantages of this method over the others include the relatively low invasiveness into the eye and the ability to secure a wide field of view. However, the stimulation charge required to induce light sensation is greater than that in the other approaches because the electrode array is far from the retina. ${ }^{(9)}$ Nonetheless, this issue may possibly be overcome by adjusting the stimulation parameters.

Biphasic symmetric pulses with charge balancing as electrical stimulation are commonly used in retinal prostheses ${ }^{(5,7,8)}$ to avoid tissue damage due to this stimulation. ${ }^{(10)}$ It has been reported that biphasic charge-balanced symmetric pulses are necessary to prevent electrode degradation due to electrochemical irreversible reactions on the electrode surface. ${ }^{(10)}$ Biphasic pulses are also used in cochlear implants. In cochlear implants, it has been reported that the threshold is reduced by setting the stimulus waveform shape of a biphasic asymmetric pulse in a state where the charge balance is maintained. ${ }^{(11)}$ However, the effect of an asymmetric pulse on an artificial retina is unknown. If a response can be obtained with a lower level of charge, the spread of the stimulation can be limited. Therefore, this study was performed to determine whether an asymmetric pulse is effective in STS.

\section{Materials and Methods}

\subsection{Animals}

We used normal rats (Long-Evans; $n=9$ ) and retina-degenerated rats (Royal College of Surgeons, RCS; $n=6$ ), aged 8-11 weeks and 20-23 weeks, respectively. All procedures were in accordance with the Association for Research in Vision and Ophthalmology Statement for the Use of Animals in Ophthalmic and Vision Research. This study was approved by the Institutional Animal Care Committee of Nidek Co., Ltd. 


\subsection{Surgical preparation}

All animals were anesthetized for surgery and acute electrophysiological recordings by intraperitoneal injection of urethane $(1.75 \mathrm{~g} / \mathrm{kg})$. Their heads were fixed to a stereotaxic apparatus (SR-5R-HT; Narishige Science Institute Laboratory, Tokyo, Japan). The dorsal surface of the left eyeball was exposed, and the small area of the exposed sclera, from approximately 1 $\mathrm{mm}$ below the optic nerve to the vicinity of the equator, was semiresected to place a platinum stimulating electrode (diameter, $0.5 \mathrm{~mm}$; height, $0.3 \mathrm{~mm}$ ) attached to a micromanipulator (SF-1; $\mathrm{S}$, Tokyo, Japan). Mineral oil was used around the eyeball to prevent drying. A return electrode (22-G needle) was positioned in the subcutaneous tissue of the nose. Craniotomy in the right parietal bone was performed, and the cortex was gently removed with the aspirator (Aspirator MINIC W-II; Shin-Ei Industries, Saitama, Japan) to expose the dorsal surface of the superior colliculus (SC) entirely. Mineral oil was applied to the exposed SC area during the experiment to prevent drying. A stainless-steel screw was inserted into the cerebellum as the reference electrode.

\subsection{Photic stimulation}

Photic stimulation was performed in all rats to confirm the retinal degeneration of RCS rats. Their left eyes were dilated with a mydriatic agent (Mydrin-P ophthalmic solution; Santen Pharmaceutical, Osaka, Japan) and the corneas were covered with a contact lens electrode combined with LED (R250 LEDT; Mayo, Aichi, Japan). After dark adaptation for 20 min in a dark room, photic stimulation (luminance, $1000 \mathrm{~cd} / \mathrm{m}^{2}$; duration, $1 \mathrm{~ms}$; frequency, $1 \mathrm{~Hz}$ ) was delivered to the eyes through the LED using a light-emitting device (LS-100; Mayo).

\subsection{Electrical stimulation}

Electrical stimulation was performed after photic stimulation in all rats. Figure 1 shows the setup for electrical stimulation. A single biphasic current pulse was applied between the

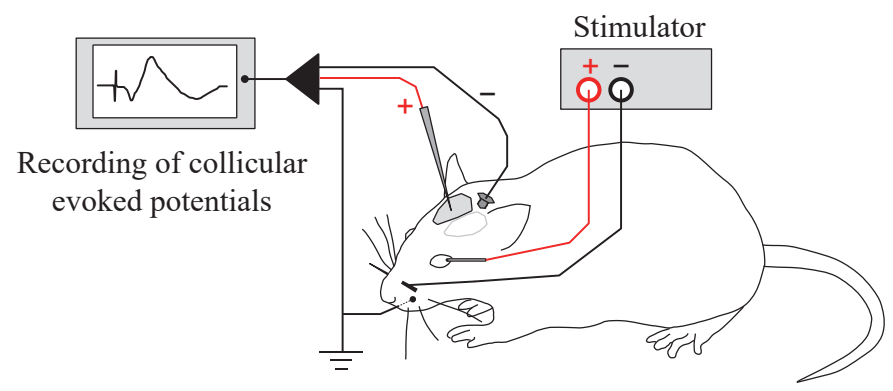

Fig. 1. (Color online) Setup for electrical stimulation and recording. A stimulating electrode $(+)$ and a return electrode (-) were positioned in the partial scleral resection of the eyeball and in the subcutaneous tissue of the nose, respectively. A single biphasic current pulse (outward current/cathodic first) was applied between these electrodes using a stimulator. The potentials evoked by electrical stimulation were recorded with a needle electrode in the SC. 
stimulating electrode and the return electrode using a stimulus generator (STG 4002; Multi Channel Systems, Reutlingen, Germany). In this study, from a practical point of view, only charge-balanced biphasic pulses were used. The amplitude and pulse duration of the biphasic pulse in the cathodal phase were fixed, and only the amplitude and pulse duration of the anodal phase were changed. The current pulses were biphasic symmetric and asymmetric pulses (cathodic first), and the stimulation frequency was $1 \mathrm{~Hz}$. The pulse duration of the cathodal phase was 0.5 $\mathrm{ms}$, and the current amplitude was $100 \mu \mathrm{A}$. The pulse duration of the anodal phase was varied from 0.2 to $2.0 \mathrm{~ms}$. Charge balances were adjusted by changing the amplitude of the electrical current (Fig. 2).

\subsection{Electrophysiological recordings}

A tungsten microelectrode [resistance, $0.5 \mathrm{M} \Omega$; diameter, approximately $5.0 \mu \mathrm{m}$ (uninsulated part); Unique Medical, Tokyo, Japan] was inserted to a depth of $250 \mu \mathrm{m}$ from the surface of the SC using a micromanipulator (MMO-203; Narishige). The evoked potentials (EPs) were amplified with a differential amplifier (DP-301, Warner, Connecticut, USA) (amplification: photic stimulation, 100 times; electrical stimulation, 1000 times; band-pass filter, $10 \mathrm{~Hz}-3$ $\mathrm{kHz}$ ) and then digitally sampled and averaged by PowerLab [PowerLab 2/26 (ML 826); ADInstruments, Dunedin, New Zealand] using software for data acquisition (LabChart, ADInstruments). The sampling frequency was set at $10 \mathrm{kHz}$ for both photic and electrical stimulations. The average signals used for both normal and RCS rats were 300 photic stimulations and 30 electrical stimulations. The position of the largest electrical EPs was determined by moving the recording electrode horizontally or vertically from the midline at intervals of 100-250 $\mu \mathrm{m}$. Subsequent recordings were completed at this position.

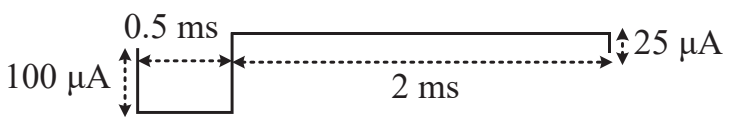

(a)

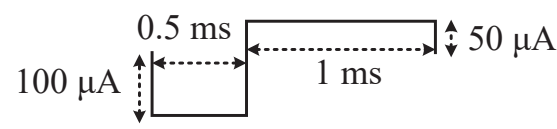

(b)

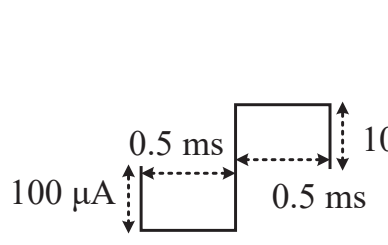

(c)

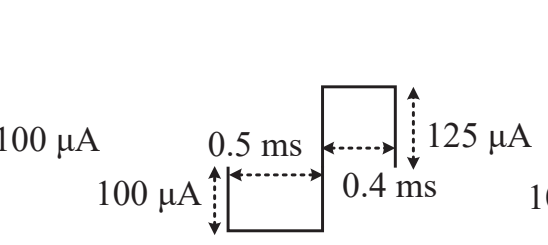

(d)

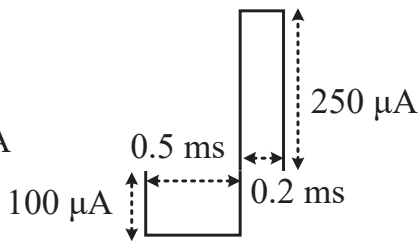

(e)

Fig. 2. Waveforms of electrical stimulation: durations of (a) 0.5:2.0, (b) 0.5:1.0, (c) 0.5:0.5, (d) 0.5:0.4, and (e) 0.5:0.2 $\mathrm{ms}$. The current pulses were biphasic symmetric and asymmetric pulses (cathodic first). The pulse duration of the cathodal phase was $0.5 \mathrm{~ms}$, and the current amplitude was $100 \mu \mathrm{A}$. The pulse duration of the anodal phase varied from 0.2 to $2.0 \mathrm{~ms}$. Charge balances were adjusted by changing the amplitude of the electrical current. 


\subsection{Data analysis}

To evaluate the waveforms of the averaged EPs, we used the difference $(\mathrm{P} 1-\mathrm{N} 1)$ between the first positive peak (P1) and the subsequent first negative peak (N1) at approximately 5 and $10 \mathrm{~ms}$ after stimulation. Python $3^{(12)}$ was used to calculate the values of P1 and N1. By changing the pulse duration and current amplitude of the anodal phase and making it an asymmetric pulse, the paired t-test was applicable to analyze whether there was a difference in the amplitude of the EP. The significance level was set at $p<0.05$. R version 3.3.2 ${ }^{(13)}$ was used for the statistical analysis.

\subsection{Histological analysis}

After completion of the experiment, all rats were euthanized with an overdose of sodium pentobarbital (150 mg/kg, i.p., Somnopentyl; Kyoritsu Seiyaku, Tokyo, Japan). From two normal rats and three RCS rats randomly selected for histological analysis, the eyes were extracted immediately after euthanasia. The extracted eyeballs were fixed for $24 \mathrm{~h}$ with a solution of $1.5 \%$ glutaraldehyde (Kanto Chemical, Tokyo, Japan) and 3\% formaldehyde (SigmaAldrich, Missouri, USA) in phosphate buffer (1/15 M, pH 7.4). Next, the globes were postfixed with a neutral buffered formalin solution for 5-7 d. After embedding in resin, the eyeballs were cut into sections of 4-5 $\mu \mathrm{m}$ thickness (Sept Sapie, Tokyo, Japan). The tissue sections were stained with hematoxylin and eosin (HE) (Sept Sapie) and then observed under a microscope (E1000M; Nikon, Tokyo, Japan) to examine the retinal cytoarchitecture.

\section{Results}

\subsection{Response waveform}

Examples of the response waveforms for photic and electrical stimulations are shown (Figs. 3 and 4). The same waveform was obtained for all rats. Approximately 30-200 ms after photic stimulation, clear EPs composed of several waves were recorded in all normal rats [Fig. 3(a)]. Meanwhile, EPs after photic stimulation were not obtained in any RCS rat [Fig. 3(b)], suggesting that the photoreceptors had degenerated severely. EPs for the electrical stimulation were composed of the first positive wave and the subsequent first negative wave approximately 5 and $10 \mathrm{~ms}$ after stimulation, respectively, in all normal and RCS rats (Fig. 4). The former and the latter were defined as P1 and N1, respectively, after the previous study (Kanda et al.). ${ }^{(14)}$ Latencies of P1 and N1 were slightly slower in RCS rats than in normal rats under all stimulation conditions.

\subsection{Effect of asymmetric electrical pulse}

In most cases, upon stimulation with an asymmetric pulse with an anodal phase of $0.2 \mathrm{~ms}$, a negative peak, N2, was confirmed at $15-25 \mathrm{~ms}$ of the response waveform in normal rats and 


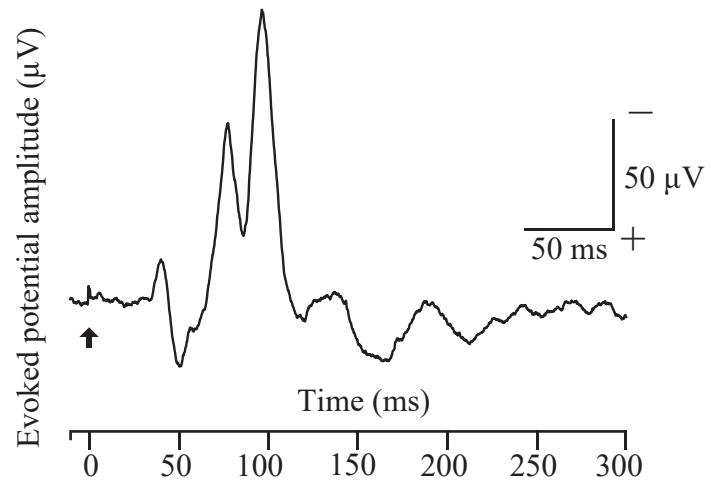

(a)

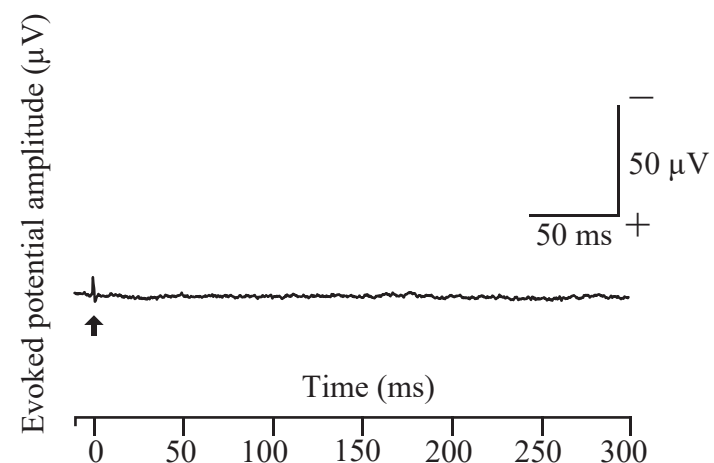

(b)

Fig. 3. An example of EPs in response to photic stimulation in (a) a normal rat (Long-Evans) and (b) a RCS rat. Artifacts that appeared when a flashlight was irradiated are indicated by arrows.

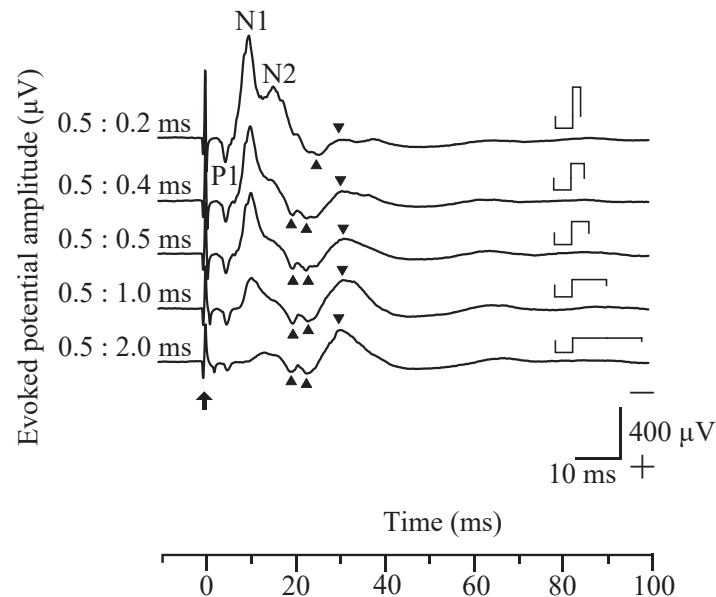

(a)

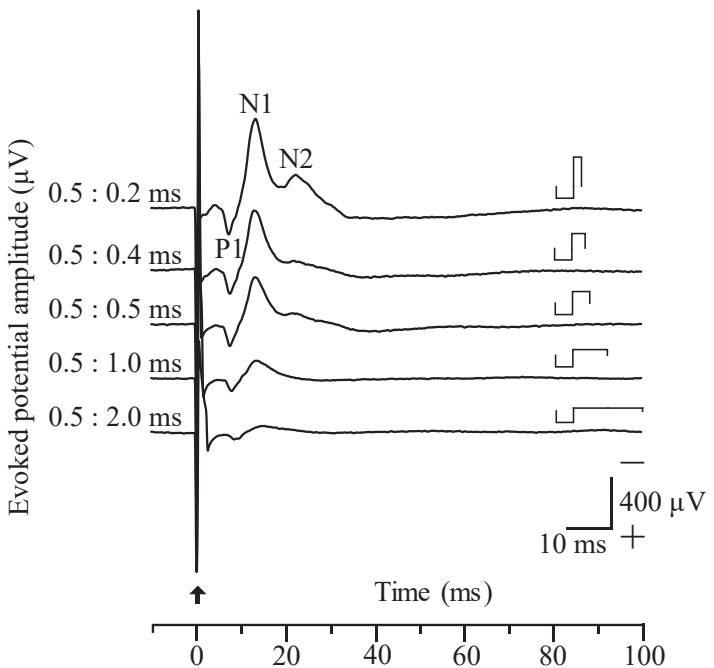

(b)

Fig. 4. An example of the response waveforms observed after electrical stimulation in (a) normal rats (LongEvans) and (b) RCS rats. The artifacts of electrical stimulation, indicated by arrows, show the application of the current pulse. In most cases, artifacts appeared larger in RCS rats than in normal rats. EPs were composed of the first positive wave and the subsequent negative wave at approximately 5 and $10 \mathrm{~ms}$ after stimulation, respectively, in all rats. In most cases, upon stimulation with an asymmetric pulse with an anodal phase of $0.2 \mathrm{~ms}$, a negative peak, $\mathrm{N} 2$, was confirmed at $15-25 \mathrm{~ms}$ in the response waveform in normal rats and RCS rats. N2 was not observed in any rat under other stimulation conditions. In all normal rats, we could confirm the positive peak at approximately 20 $\mathrm{ms}$ and the negative peak at approximately $30 \mathrm{~ms}$ (the peaks are indicated by triangles).

RCS rats (Fig. 4). Under other stimulation conditions, N2 disappeared in all normal rats and RCS rats. P1 and N1 were also observed upon application of an asymmetric pulse with the anodal phase having a longer pulse duration than the cathodal phase, but the heights of P1 and $\mathrm{N} 1$ decreased as the pulse duration of the anodal phase became longer, and the peaks became 
less well defined (Fig. 4). For all normal rats, positive and negative peaks were observed at approximately 20 and $30 \mathrm{~ms}$ after stimulation, respectively. These peaks increased with an increase in the pulse duration of the anodal phase [indicated by triangles in Fig. 4(a)]. However, peaks beyond $20 \mathrm{~ms}$ after stimulation could not be obtained in any RCS rat [Fig. 4(b)].

The EP amplitudes (P1 - N1) are shown in Fig. 5. In both normal rats and RCS rats, the pulse duration of the anodal phase was shorter and, as the current amplitude increased, the EP amplitude also increased (Fig. 5).

\subsection{Histological analysis}

Figure 6 shows the typical microscopy images of HE-stained retinal sections. In RCS rats, the layered structures of the outer retina had degenerated [Fig. 6(b)]. In the three RCS rats for which the tissues were observed, the presence of the outer retinal layers could not be confirmed, whereas the structures from the inner nuclear layer to the ganglion cell layer were evident. The two normal rats showed intact inner and outer retinal layers [Fig. 6(a)].

\section{Discussion}

None of the RCS rats used for this study responded to photic stimulus. Morphologically, it has been reported that outer nuclear layer degeneration starts when the rats are aged around $30 \mathrm{~d}$, and it completely degenerates by age $104 \mathrm{~d}^{(15,16)}$ Therefore, it can be inferred that RCS rats in this study had a sufficiently advanced degeneration of photoreceptors. With electrical stimulation, the response peaks of positive and negative waves, typically observed at

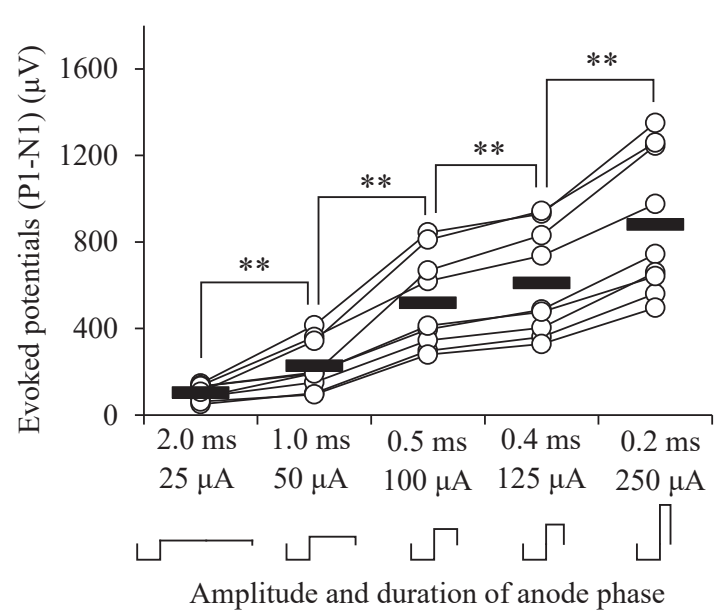

(a)

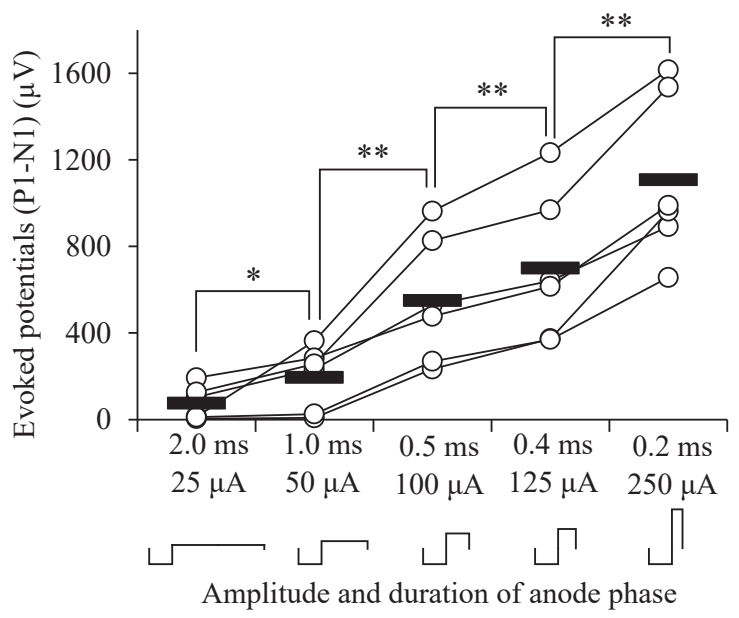

(b)

Fig. 5. EP amplitudes after electrical stimulation in (a) normal rats (Long-Evans) $(n=9)$ and (b) RCS rats (n $=6$ ). The black bars (-) represent each mean, and the circle (o) represents the value for each animal. The lines connecting the symbols (o) indicate that the data are from the same individual. Paired t-test $\left({ }^{*} p<0.05 ;{ }^{* *} p<0.01\right)$ was used to detect significant differences. As the pulse duration of the anodal phase became shorter and the current amplitude became higher, the EP amplitude was significantly greater $(p<0.05)$. 


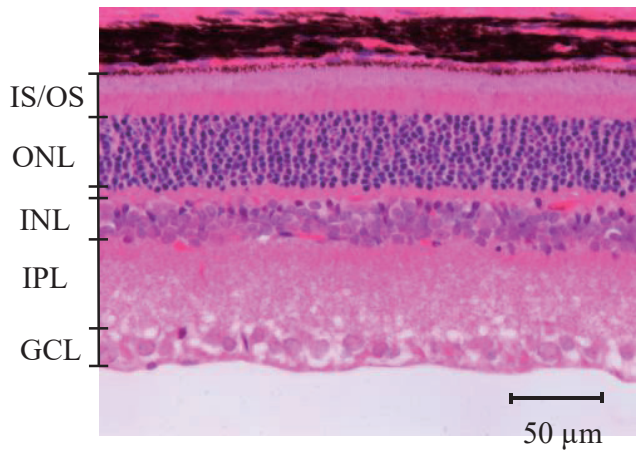

(a)

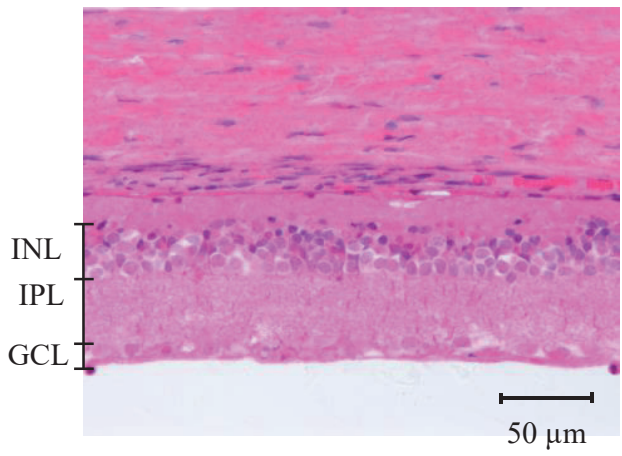

(b)

Fig. 6. (Color online) Representative tissue specimens of (a) normal 10-week-old rats (Long-Evans) and (b) 21-week-old RCS rats. The sclae bars represent $50 \mu \mathrm{m}$. The inner segment/outer segment (IS/OS), outer nuclear layer (ONL), inner nuclear layer (INL), inner plexiform layer (IPL), and ganglion cell layer (GCL) are indicated. The outer retina was lost in RCS rats.

approximately 20 and $30 \mathrm{~ms}$ after stimulation in normal rats [indicated by triangles in Fig. 4(a)], could not be obtained with RCS rats [Fig. 4(b)]. Therefore, it can be inferred that the response peaks after $20 \mathrm{~ms}$ are responses from a part of the retina, such as photoreceptor cells, that does not function in the degenerated retina of RCS rats. In addition, the amplitude of the response peaks after $20 \mathrm{~ms}$ increased as the pulse duration of the anodal phase became longer in normal rats [Fig. 4(a)]. This is in agreement with previous results that pulse durations longer than 0.5 ms were effective for the stimulation of retinal cells outside bipolar cells (such as photoreceptor cells). ${ }^{(17)}$ In contrast, response peaks P1 and N1 were obtained in both normal rats and RCS rats after photoreceptor cell degeneration. This is the reason why we defined EP amplitude as the difference between P1 and N1. Latencies of P1 and N1 were slower in RCS rats than in normal rats. Similar results were previously reported by Kanda et al. ${ }^{(14)}$ Several previous studies have revealed some abnormalities in horizontal, Müller, and bipolar cells of the retina of RCS rats. ${ }^{(18-21)}$ These abnormalities may delay the latencies of P1 and N1 in RCS rats. For the delay of latency in RCS rats, further detailed study will be necessary.

The purpose of this study was to determine whether an asymmetric current waveform is effective in STS. In this study, from a practical point of view, only the charge-balanced biphasic pulse was used. Therefore, for example, if the current amplitude in the anodal phase of the symmetric pulse is increased keeping the charge per phase constant, the duration of the anodal phase is shortened. In this study, we focused on the effect of the anodal phase; therefore, the amplitude and duration of the cathodal phase were kept constant. Accordingly, biphasic pulses with modulated amplitude of the anodal phase had asymmetric shapes (Fig. 2). Our results suggested that a charge-balanced biphasic asymmetric pulse with a short pulse duration and high current amplitude in the anodal phase has better stimulation efficiency on the retina than a symmetric pulse with the same electrical charge (Fig. 5). Additionally, as the pulse duration of the anodal phase decreased and as the current amplitude increased, the EP amplitude increased (Fig. 5). It remains unclear whether the amplitude or the duration of the anodal phase played 
the dominant role in these phenomena. The results of previous studies suggest that both the duration and the amplitude affect the magnitude of the neural response. ${ }^{(14,22)}$ In this study, both the pulse duration and the current amplitude of the cathodal phase were kept constant because we focused on the efficacy of the anodal phase. Therefore, even with a symmetric pulse with shorter pulse duration and higher current amplitude of both the anodal phase and the cathodal phase, there is a possibility of obtaining the same result as that with an asymmetric pulse of shorter pulse duration and higher current amplitude. Symmetrical pulses with shorter pulse duration and higher current amplitude of both the anodal phase and the cathodal phase will be studied further in the future. In the following sentence, "asymmetric pulse" refers to the pulse with shortened pulse duration and increased current amplitude used in this study.

Although we did not measure the threshold charge, our results showed that the abovedescribed asymmetric pulse with shorter pulse duration and higher current amplitude in the anodal phase evoked a larger neural response. This suggested that an asymmetric pulse, with shorter pulse duration and higher current amplitude in the anodal phase, can induce a response with smaller electrical charges than a symmetric pulse. In the future, to improve the spatial resolution when developing a smaller electrode for retinal prostheses, it is necessary to stimulate the retina without increasing the charge density, in order to avoid electrode deterioration and tissue damage. ${ }^{(10)}$ Our results suggest that asymmetric pulses with shorter pulse duration and higher current amplitude in the anodal phase evoke neural responses with less charge than symmetric pulses. Accordingly, by using such an asymmetric pulse (as described previously), electrical stimulation becomes feasible without increasing the charge density, even when the electrode area is reduced. Sekirnjak et al. reported that reducing the electrode area can limit the range of stimulated retinal ganglion cells to a spatially confined region. ${ }^{(23)}$ Therefore, the excitation of the retina is localized by reducing the electrode area, and the excitement of the visual cortex can also be confined. As a result, the phosphene, which is a pseudolight sensation by electrical stimulation, can be expected to localize.

Currently, the STS has 49 electrodes in the electrode array. Cathodic-first biphasic pulses of $0.5 \mathrm{~ms}$ duration are sequentially delivered to individual electrodes. ${ }^{(8)}$ However, in the present system, it is not possible to stimulate multiple electrodes simultaneously. Thus, each electrode is sequentially stimulated. The frame rate used in the previous STS clinical trial was $20 \mathrm{~Hz} .{ }^{(8)}$ If we increase the number of electrodes, the stimulation frequency would be very low because at least $49 \mathrm{~ms}$ is necessary to apply current pulses to all 49 electrodes (this corresponds to approximately $20 \mathrm{~Hz}$ ). For example, if the number of electrodes increases to 100, the frequency becomes $10 \mathrm{~Hz}$. The pulses with shorter pulse duration and higher current amplitude in the anodal phase used in this study can reduce the pulse duration without sacrificing neural excitability. Thus, the above-described asymmetric pulse has the potential to reduce this limitation.

In this study, we have not confirmed the efficacy of asymmetric pulses with different pulse durations and current amplitudes in the cathodal phase. Therefore, the effect of an asymmetric pulse when changing the amplitude and the duration of the cathodal phase is unknown. This will be examined in future work. Moreover, we focused on cathodic-first stimulation in this study because we are using cathodic-first stimulation in clinical trials for the retinal prosthesis 
that we are developing. ${ }^{(8)}$ However, we also consider anodic-first biphasic stimulation to be important and it will be studied further in the future.

This study is the first to show the effectiveness of an asymmetric pulse with a short pulse duration and high current amplitude in the anodal phase in vivo for STS. We also confirmed that all RCS rats in this study exhibited photoreceptor cell degeneration [Figs. 3(b) and 6(b)]. RCS rats are animal models of the RP; however further studies are needed to determine whether the results obtained using RCS rats are applicable to RP patients.

\section{Conclusions}

We confirmed that a charge-balanced biphasic asymmetric pulse with a short pulse duration and high current amplitude in the anodal phase could stimulate the retina more efficiently than a symmetric pulse with the same electrical charge. Because asymmetric pulses with a short pulse duration and high current amplitude in the anodal phase were suggested to reduce the electrical charge to evoke neural response, surface area of the stimulating electrode, corresponding to the electrical charge, could be reduced. Therefore, there is the possibility of localizing the phosphenes by limiting the stimulated retinal cells to a certain region. Consequently, by using an asymmetric pulse with a short pulse duration and high current amplitude in the anodal phase, high-resolution retinal prostheses can be expected. Our results suggest that an asymmetric pulse with a short pulse duration and high current amplitude in the anodal phase is effective for retinal prostheses.

\section{References}

1 H. Yokoi, K. Sato, S. Morishita, T. Nakamura, R. Kato, T. Umeda, H. Watanabe, Y. Nishimura, T. Isa, K. Ikoma, T. Miyamoto, and O. Yamamura: Advances in Therapeutic Engineering, ed. W. Yu (CRC Press, Boca Raton, 2012) p. 219.

2 M. Hirata, T. Yanagisawa, K. Matsushita, H. Sugata, Y. Kamitani, T. Suzuki, H. Yokoi, T. Goto, M. Shayne, Y. Saitoh, H. Kishima, M. Kawato, and T. Yoshimine: Technological Advancements in Biomedicine for Healthcare Applications, ed. J. Wu (Medical Information Science Reference, Hershey, 2012) p. 362.

3 NIDCD Information Clearinghouse: NIDCD Fact Sheet Cochlear Implants, NIH Publication No. 11-4798 (2016).

4 D. T. Hartong, E. L. Berson, and T. P. Dryja: The Lancet 368 (2006) 1795.

5 D. H. Ghodasra, A. Chen, J. F. Arevalo, D. G. Birch, K. Branham, B. Coley, G. Dagnelie, E. de Juan, R. G. Devenyi, J. D. Dorn, A. Fisher, D. R. Geruschat, N. Z. Gregori, R. J. Greenberg, P. Hahn, A. C. Ho, A. Howson, S. S. Huang, R. Iezzi, N. Khan, B. L. Lam, J. I. Lim, K. G. Locke, M. Markowitz, A.-M. Ripley, M. Rankin, H. Schimitzek, F. Tripp, J. D. Weiland, J. Yan, D. N. Zacks, and K. T. Jayasundera: BMC Ophthalmol. 16 (2016) 1.

6 K. Stingl, K. U. Bartz-Schmidt, D. Besch, C. K. Chee, C. L. Cottriall, F. Gekeler, M. Groppe, T. L. Jackson, R. E. MacLaren, A. Koitschev, A. Kusnyerik, J. Neffendorf, J. Nemeth, M. A. Naeem, T. Peters, J. D. Ramsden, H. Sachs, A. Simpson, M. S. Singh, B. Wilhelm, D. Wong, and E. Zrenner: Vision Res. 111 (2015) 149.

7 N. C. Sinclair, M. N. Shivdasani, T. Perera, L. N. Gillespie, H. J. McDermott, L. N. Ayton, and P. J. Blamey: Invest. Ophthalmol. Vis. Sci. 57 (2016) 4948.

8 T. Fujikado, M. Kamei, H. Sakaguchi, H. Kanda, T. Endo, M. Hirota, T. Morimoto, K. Nishida, H. Kishima, Y. Terasawa, K. Oosawa, M. Ozawa, and K. Nishida: Invest. Ophthalmol. Vis. Sci. 57 (2016) 6147.

9 T. Morimoto, M. Kamei, K. Nishida, H. Sakaguchi, H. Kanda, Y. Ikuno, H. Kishima, T. Maruo, K. Konoma, M. Ozawa, K. Nishida, and T. Fujikado: Invest. Ophthalmol. Vis. Sci. 52 (2011) 6785.

10 S. F. Cogan: Annu. Rev. Biomed. Eng. 10 (2008) 275.

11 O. Macherey, A. V. Wieringen, R. P. Carlyon, J. M. Deeks, and J. Wouters: J. Assoc. Res. Otolaryngol. 7 (2006) 253.

12 Python Software Foundation: Python, https://www.python.org/ (accessed November 2016).

13 R Core Team: The R Project for Statistical Computing, https://www.r-project.org/ (accessed November 2016). 
14 H. Kanda, T. Morimoto, T. Fujikado, Y. Tano, Y. Fukuda, and H. Sawai: Invest. Ophthalmol. Vis. Sci. 45 (2004) 560.

15 R. C. Ryals, M. D. Andrews, S. Datta, A. S. Coyner, C. M. Fischer, Y. Wen, M. E. Pennesi, and T. J. McGill: Invest. Ophthalmol. Vis. Sci. 58 (2017) 1378.

16 A. J. Eisenfeld, M. M. Lavail, and J. H. Lavail: J. Comp. Neurol. 223 (1984) 22.

17 R. J. Greenberg, M. S. Humayun, and E. de Juan Jr.: U.S. Patent No. 5944747 (1999).

18 Y. Chu, M. F. Humphrey, and I. J. Constable: Exp. Eye Res. 57 (1993) 141.

19 W. Hartig, J. Grosche, C. Distler, D. Grimm, E. El-Hifnawi, and A. Reichenbach: J. Neurocytol. 24 (1995) 507.

20 R. Hanitzsch, C. Zeumer, T. Lichtenberger, and K. Wurziger: Acta. Anat. (Basel) 162 (1998) 119.

21 Y. W. Peng, T. Senda, Y. Hao, K. Matsuno, and F. Wong: Neuroscience. 119 (2003) 813.

22 S. E. John, M. N. Shivdasani, C. E. Williams, J. W. Morley, R. K. Shepherd, G. D. Rathbone, and J. B. Fallon: J. Neural Eng. 10 (2013) 056011.

23 C. Sekirnjak, P. Hottowy, A. Sher, W. Dabrowski, A. M. Litke, and E. J. Chichilnisky: J. Neurophysiol. 95 (2006) 3311.

\section{About the Authors}

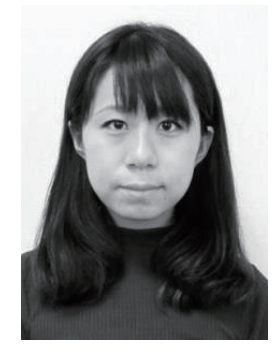

Yukari Nakano received her B.S. degree in chemical engineering from Shizuoka University, Shizuoka, Japan, in 2009. In 2009, she joined Nidek Co., Ltd., Aichi, Japan. Since 2012, she has been an investigator at the Artificial Vision Institute of Nidek Co., Ltd. Her research includes the development of active implantable devices for blind people, electrophysiological evaluation, and statistical analysis of electrophysiological evaluation.

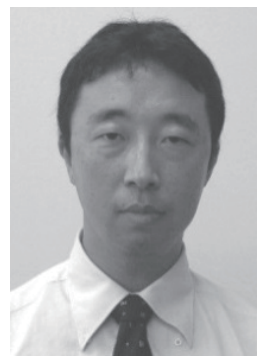

Yasuo Terasawa received his B.S. degree in applied physics and M.S. degree in information science from Tohoku University, Miyagi, Japan in 1996 and 1998, respectively. He joined Nomura Research Institute in 1998. Since 2001, he has been an investigator in the Vision Institute of Nidek Co., Ltd., Aichi, Japan. He received his Ph.D. degree in materials science in 2009 from Nara Institute of Science and Technology (NAIST), Nara, Japan. Since 2009, he has been both a research fellow in NAIST and an investigator in Nidek. Since 2016, he has been a manager of the Vision Institute of Nidek. His research includes electrode technology, microfabrication, neural interface, and implantable electronics.

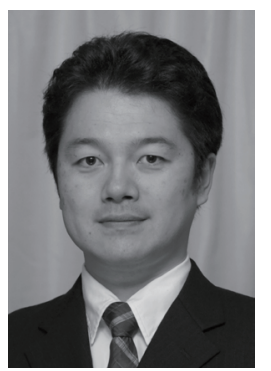

Hiroyuki Kanda received his B.S. degree from Shizuoka University, Shizuoka, Japan, in 1998, M.E. degree in computational science and engineering from Nagoya University, Nagoya, Japan, in 2000, and Ph.D degree in medicine from Osaka University, Suita, Japan, in 2005. In 2001, he joined Nidek Co., Ltd., Gamagori, Japan, where he has been engaged in research on retinal prosthesis. Since 2009, he has been an assistant professor at Osaka University, Suita, Japan. His current research interests focus on the safety and efficacy evaluation of retinal prostheses. 


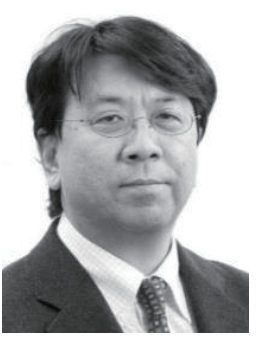

Jun Ohta received his B.E., M.E., and Dr. Eng. degrees in applied physics, all from the University of Tokyo, Japan, in 1981, 1983, and 1992, respectively. In 1983, he joined Mitsubishi Electric Corporation, Hyogo, Japan. From 1992 to 1993, he was a visiting scientist in Optoelectronics Computing Systems Center, University of Colorado at Boulder. In 1998, he joined the Graduate School of Materials Science, Nara Institute of Science and Technology, Nara, Japan, as an associate professor. He was appointed professor in 2004. His current research interests are smart CMOS image sensors for biomedical applications and retinal prosthetic devices. He serves as an Associate Editor of IEEE Sensors Journal and an Editorial Board member of The Journal of Engineering, the Institution of Engineering and Technology.

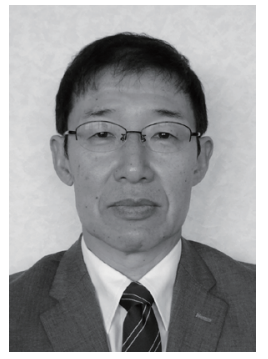

Hajime Sawai received his B.S. degree from the Faculty of Human Sciences, Osaka University, Japan, in 1982 and his M.S. and Ph.D. degrees from the Osaka University Graduate School of Medicine, Japan, in 1985 and 1992, respectively. From 1994 to 2000, he was an associate professor at Okayama Prefecture University, Japan. From 2000 to 2015, he was an associate professor at Osaka University. Since then, he has been a professor at Osaka Prefecture University. His research interests are visual neuroscience and visual prosthesis.

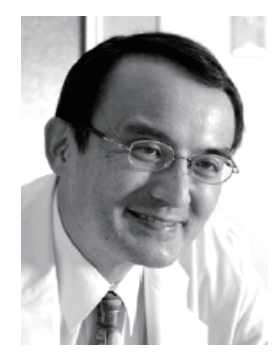

Takashi Fujikado received his M.E. degree from the University of Tokyo, Japan, in 1978 and his M.D and Ph.D. degrees from Osaka University, Japan, in 1982 and 1988, respectively. From 1992 to 1996, he was an assistant professor at Osaka University, Japan. Since 1998, he has been a professor at Osaka University. His research interests are neuro-ophthalmol, medical engineering, and BMI. 